\title{
S-13: Interventions for Prevention and Rehabilitation of Hamstring Injuries
}

\author{
Reza Rahimi Moghaddam, Mohammad Haghshenas, Maryam Asadi \\ Sports Medicine Association of Gilan Province, Iran
}

\begin{abstract}
INTRODUCTION: The hamstring muscles have very important role in the stabilization of body posture, movement of the lower extremities and trunk movements in relation to the thigh. Hamstring injuries are common among athletes, especially in sports like soccer with sprinting demands, kicking, and sudden accelerations. Hamstring strains are frustrating for the injured athletes because the symptoms are persistent, healing is slow, and the rate of re-injury is high. This indicates a need to develop prevention strategies for hamstring injuries. The aims of this review are introducing hamstring strains, associated risk factors, and providing rehabilitative ecommendations for injured athletes to prevent re-injury.

METHOD: Information was gathered from an online literatures search using the key words hamstring injuries, soccer injuries, injury prevention, hamstring rehabilitation, and stretching exercises. Screening of references and hand searches of relevant journals were also employed. All relevant studies in English were reviewed and abstracted.

RESULTS: It has been shown that hamstring strains account for $12-16 \%$ of all injuries in athletes with a re-injury rate reported as high as $22-34 \%$. The hamstrings have a tendency to shorten. Tight hamstrings with limited range of motion and flexibility may lead to postural deficiency and deformities. It also makes the hamstring susceptible to re-injury. Risk factors such as age, strength imbalance, previous injury and flexibility should be considered.

CONCLUSION: Prevention intervention may minimize the risk factors of hamstring injuries. Training modalities should emphasize on eccentric strength training, and prevention of fatigue. There is wide disagreement about the impact of stretching exercise on prevention/rehabilitation of hamstring injuries.
\end{abstract}

Available at: http://journalofsportsmedicine.org and http://dx.doi.org/10.5152/tjsm.2017.017 